\title{
Prevalência da co-infecção HIV-sífilis em um hospital universitário da Cidade do Rio de Janeiro no ano de 2005
}

\author{
Prevalence of HIV-syphilis coinfection in a university hospital \\ in the City of Rio de Janeiro in 2005
}

\author{
Dario José Hart Pontes Signorini' ${ }^{1}$, Michelle Carreira Miranda Monteiro², \\ Carlos Alberto Morais de Sá ${ }^{1}$, Fernando Samuel Sion ${ }^{1,3}$, Hilton Gueiros Leitão Neto ${ }^{2}$, \\ Dulcino Pirovani Lima² e João Dario do Carmo Machado²
}

\begin{abstract}
RESUMO
Estudo de prevalência da co-infecção HIV-sífilis realizado com 830 pacientes em acompanhamento ambulatorial para HIV/aids entre janeiro e maio de 2005 no Hospital na Cidade do Rio de Janeiro. Os participantes realizaram exames de VDRL (veneral disease research laboratory), contagens de células $C D 4+/ C D 8+$ e de carga viral e responderam perguntas sobre características sócio-demográficas e história prévia de sifilis. A prevalência da sifilis foi de 2,7\% (22), a relação entre homens e mulheres co-infectados foi de 4:1, aproximadamente. Homossexuais masculinos foram os mais acometidos e não encontramos associação entre co-infecção e idade, escolaridade e parâmetros laboratoriais testados. Do total de casos com sifilis, 73\% (10) relataram tratamento prévio; destes, 14 (88\%) pacientes foram re-infectados, enquanto 2 (12\%) pacientes realizaram tratamento inapropriado. A presença de co-infecção HIV-sífilis em pacientes em acompanhamento rotineiro alerta-nos para necessidade de aconselhá-los a adotar práticas sexuais seguras durante os seus atendimentos ambulatoriais.
\end{abstract}

Palavras-chaves: Epidemiologia. Prevalência. Sifilis. HIV/AIDS.

\section{ABSTRACT}

This was a study on the prevalence of HIV-syphilis coinfection among 830 HIV/AIDS patients who were being followed up as outpatients at a hospital in Rio de Janeiro between January and May 2005. The participants underwent laboratory tests at the venereal disease research laboratory (VDRL) consisting of CD4+/CD8+ cell counts and viral load tests. They answered questions about their sociodemographic characteristics and past medical history of syphilis. The prevalence of syphilis was 2.7\% (22 patients) and the ratio between men and women with coinfection was approximately 4:1. Homosexual men were the category most affected. We did not find any association between coinfection and age, schooling and the laboratory parameters tested. Out of the total number of patients with syphilis, $73 \%$ (16) reported previous treatment; of these, 14 (88\%) were reinfected, while two (12\%) underwent inappropriate treatment. The presence of HIV-syphilis coinfection among patients undergoing routine follow-up signals the need to advise them to adopt safe sex practices, during their outpatient visits.

Key-words: Epidemiology. Prevalence. Syphilis. HIV/AIDS.

A evolução temporal da AIDS é caracterizada por uma dinâmica de contínua transformação. Os termos juvenilização, pauperização, heterossexualização e feminilização precisaram ser incluídos no discurso sobre a epidemia para acompanhar as mudanças de seu padrão epidemiológico. A epidemia que se iniciou nos estratos sociais de maior escolaridade disseminouse progressivamente para os estratos de menor escolaridade e também dos centros urbanos para os municípios de menor populaçã $0^{67}$. Da mesma forma, a epidemia inicialmente vinculada a homens que fazem sexo com homens (HSH) tem atingido de

\footnotetext{
1. Departamento de Clínica Médica, Hospital Universitário Gaffrée e Guinle, Universidade do Rio de Janeiro (UNI-RIO), Rio de Janeiro, RJ. 2. Universidade do Rio de Janeiro, Rio de Janeiro, RJ. 3. Laboratório de Pesquisa em Imunologia e AIDS, Hospital Universitário Gaffrée e Guinle, Universidade do Rio de Janeiro, Rio de Janeiro, RJ.

Endereço para correspondência: Dr. Dario José Hart Pontes Signorini. Dept ${ }^{0}$ de Clínica Médica/Hosp Univ Gaffrée e Guinle/UNI-RIO. Av. Nossa Senhora de Copacabana 709/1207, Copacabana, 22050-000 Rio de Janeiro, RJ.

e-mail: dariohart@terra.com.br

Recebido para publicação em 14/11/2006

Aceito em 26/4/2007
} 
forma crescente homens com comportamento heterossexual, mulheres e crianças ${ }^{14}$.

A elevada prevalência de outras doenças sexualmente transmissíveis (DST), como a sífilis, cuja prevalência no Brasil é estimada em 1 milhão de $\operatorname{casos}^{12}$ e a associação da prática sexual de risco na transmissão do vírus da imunodeficiência humana (HIV) têm sugerido a importância da obtenção de dados epidemiológicos em diferentes grupos populacionais a fim de orientar medidas de saúde pública a serem tomadas de forma mais apropriada.

Pessoas infectadas pelo HIV apresentam taxas mais altas de infecção por outras DST. Isso pode ocorrer devido a fatores comportamentais, biológicos, como o aumento da suscetibilidade às DST ou consequiência do estado de imunossupressão ou até mesmo resultar da interação de ambos os fatores5.

Nos anos 90, houve diminuição da incidência da sífilis nos Estados Unidos ${ }^{8}$ cuja razão, ainda que não totalmente compreendida, estaria relacionada às medidas de controle da sífilis e prevenção da $\operatorname{AIDS}^{2}{ }^{8}$; mudanças nas práticas sexuais devido à epidemia da $\operatorname{AIDS}^{7}$; a imunidade adquirida para sífilis da população em risco ${ }^{2}{ }^{8}$ e declínio do uso de crack e de cocaína no final dos anos $80^{2}$. Similarmente, as altas taxas de mortalidade da aids nesse mesmo país e período, reduziram o número de indivíduos susceptíveis às DST, especificamente a sífilis. Dessa forma, a epidemia da infecção HIV contribuiu diretamente para declínio das taxas de incidência da sífilis ${ }^{5}$.

Estudos mais recentes sobre a co-infecção HIV-sífilis têm mostrado que a sífilis é a principal DST associada ao HIV, especialmente em HSH. Em estudo que estimou a taxa de incidência de síflis primária e secundária nos EUA no ano de 2002, foi observada uma taxa de incidência de sífilis, em indivíduos infectados pelo HIV, de 186/100.000, enquanto na população em geral essa mesma taxa foi estimada em 2,4/100.000. Cerca de $1 / 4$ dos casos de sífilis ocorreu em portadores do HIV 5 .

Pelos motivos descritos anteriormente tem sido recomendado um rastreamento regular para DST em indivíduos infectados pelo HIV em acompanhamento clínico ${ }^{13}$. Em função da sífilis e do HIV compartilharem os mesmos fatores de risco, a avaliação do perfil epidemiológico da co-infecção HIV-sífilis é necessária para orientar estratégias de intervenção mais adequadas. 0 objetivo deste estudo foi avaliar a prevalência da infecção pela sífilis nos pacientes portadores do HIV e analisar características sócio-demográficas, clínicas e comportamentais associadas a essa co-infecção HIV-sífilis.

\section{MATERIAL E MÉTODOS}

Foi realizado um estudo transversal em 830 pacientes infectados pelo HIV/AIDS, com idade mínima de 13 anos, em acompanhamento ambulatorial no Hospital Universitário na Cidade do Rio de Janeiro, entre janeiro a maio de 2005. Todos os pacientes participantes do estudo assinaram o termo de consentimento e realizaram os exames VDRL (Veneral Disease Research Laboratory), contagem de células CD4+, CD8+ e quantificação da carga viral, além de responderem a um questionário onde se averiguou sexo, idade, grau de escolaridade, categoria de exposição e história prévia de sífilis. 0 estudo foi aprovado pelo comitê de ética em pesquisa da instituição.

0 diagnóstico da sífilis foi realizado através do resultado da titulação $\geq 1 / 16$ do VDRL, titulações menores foram tratadas como resultado falso positivos.

A análise exploratória dos dados foi feita no pacote estatístico $\mathrm{R}^{17}$. Utilizou-se para compararem médias e proporções entre os grupos os testes T de Student e o teste qui-quadrado, para um nível significância estatística de 5\%, respectivamente. As estimativas odds ratio $(\mathrm{OR})$ dos fatores determinantes para o diagnóstico da sífilis foram feitas através de análise de regressão logística, utilizando sexo (homens/mulheres), categoria de exposição (homossexuais/outras categorias) e idade (contínua), como, variáveis independentes. 0 teste Hosmer - Lemeshow foi realizado para medir o ajuste (goodness-of-fit) do modelo de regressão logística.

\section{RESULTADOS}

Do total de 830 pacientes, 377 eram do sexo feminino e 453 do sexo masculino. A idade mínima foi de 13 anos, e a máxima de 75 anos, sendo a média e mediana de idade 41 anos e 40 anos, respectivamente. Em relação ao grau de escolaridade da população em estudo, 1\% era de analfabetos, 33,2\% possuíam ensino fundamental incompleto, 16,6\% fundamental completo, 11,8\% médio incompleto, $25,7 \%$ médio completo, e 11,7\% superior.

Dentre os participantes do estudo, 77,4\% (642) nunca haviam sido tratados previamente para a infecção pela sífilis, enquanto 21,4\% (178) já haviam recebido tratamento prévio e 1,2\% (10) não sabiam informar.

Dos 830 exames de VDRL realizados, houve uma perda de 8 , devido a extravio do resultado pelo laboratório, sendo o total de indivíduos estudados para co-infecção HIV-sífilis de 822 pacientes. A Tabela 1 mostra os resultados dos exames de VDRL.

Tabela 1 - Freqüiência dos títulos de VDRL encontrados nos 822 pacientes portadores do HIV/AIDS de um hospital público da Cidade do Rio de Janeiro em 2005.

\begin{tabular}{lrr}
\hline & \multicolumn{2}{c}{ Pacientes } \\
\cline { 2 - 3 } VDRL & $\mathrm{n}^{0}$ & $\%$ \\
\hline Negativo & 696 & 84,7 \\
$1 / 1$ & 38 & 4,6 \\
$1 / 2$ & 25 & 3,0 \\
$1 / 4$ & 22 & 2,7 \\
$1 / 6$ & 1 & 0,1 \\
$1 / 8$ & 17 & 2,1 \\
$1 / 12$ & 1 & 0,1 \\
$1 / 16$ & 10 & 1,2 \\
$1 / 32$ & 4 & 0,5 \\
$1 / 64$ & 4 & 0,5 \\
$1 / 128$ & 2 & 0,2 \\
$1 / 256$ & 2 & 0,2 \\
\hline
\end{tabular}

VDRL: veneral disease research laboratory 
A prevalência da sífilis na amostra foi de $2,7 \%$ (22). Entre os homens a proporção foi de $4 \%$ (18) e entre as mulheres de 1,1\% (4). A Tabela 2 mostra a distribuição dos casos de sífilis por sexo encontrado na amostra. Dos 22 pacientes com sífilis, $16(72,7 \%)$ relataram tratamento prévio, enquanto $6(27,3 \%)$ não relataram. Dos 16 pacientes previamente tratados, 14 obtiveram cura com o tratamento anterior e apresentavam nova infecção e 2 tinham realizado o tratamento anterior de forma incorreta.

Tabela 2 - Freqüência dos títulos de VDRL encontrados nos pacientes portadores do HIV/AIDS de um hospital público da Cidade do Rio de janeiro em 2005, segundo o gênero.

\begin{tabular}{lrrrrr}
\hline & \multicolumn{4}{c}{ Sexo } \\
\cline { 2 - 3 } Resultado do VDRL & \multicolumn{2}{c}{ feminino } & & \multicolumn{2}{c}{ masculino } \\
\cline { 2 - 3 } \cline { 6 - 7 } & $\mathrm{n}^{0}$ & & & $\mathrm{n}^{0}$ & $\%$ \\
\hline Positivo (título $\geq 1 / 16)$ & 4 & 1,1 & & 18 & 4,2 \\
Negativo (título $\leq 1 / 8)$ & 372 & 98,9 & & 428 & 95,8 \\
\hline
\end{tabular}

VDRL: veneral disease research laboratory

Os homens apresentaram mais infecção por sífilis que as mulheres e essa diferença foi estatisticamente significativa $(\mathrm{p}<0,01)$. Quando foi estudada a relação entre categoria de exposição e resultado do VDRL observamos que a infecção por sífilis foi mais freqüente entre os homossexuais masculinos $(\mathrm{p}<0,000)$.

Não houve diferença significativa em relação à escolaridade quanto a apresentar infecção por sífilis ou não $(\mathrm{p}=0,14)$. Também, não houve associação entre positividade no VDRL e na contagem de CD4, CD8 e carga viral ( $p=0,53 ; 0,94$ e 0,82, respectivamente). A infecção por sífilis também não esteve associada à idade $(\mathrm{p}=0,14)$.

$\mathrm{Na}$ análise multivariada, o diagnóstico da sífilis foi, aproximadamente, 3 vezes mais comum entre os homossexuais masculinos do que nas demais categorias $(p=0,03)$, já as estimativas de OR no modelo de regressão logística para sexo e idade não apresentaram significância estatística (Tabela 3).

Tabela 3 - Análise multivariada dos fatores determinantes associados ao diagnóstico da sífilis nos 822 pacientes portadores do HIVIAIDS de um hospital público da Cidade do Rio de Janeiro em 2005.

\begin{tabular}{lccc}
\hline Variáveis & Categorias & 95\% C.I. (OR Lower- Upper) & p- valor \\
\hline Sexo & $\begin{array}{c}\text { mulheres* } \\
\text { homens }\end{array}$ & $2,5(0,71-8,5)$ & 0,15 \\
\hline Categoria de & $\begin{array}{c}\text { Demais categorias* } \\
\text { Homossexuais }\end{array}$ & $2,9(1,1-7,7)$ & 0,03 \\
exposição & & $0,97(0,93-1,01)$ & 0,18 \\
\hline Idade & & &
\end{tabular}

$\mathrm{n}=822$ casos

* categoria de referência

Teste de Hosmer e Lemeshow: $\mathrm{p}=.55$

\section{DISCUSSÃO}

A proporção de co-infectados encontrados em nosso estudo foi menor do que aquelas encontradas em outros ${ }^{913}$ que apresentaram respectivamente 7,8\% e 8,8\%. Aventamos que essas diferenças de prevalência sejam decorrentes, não só, da origem de cada população em estudo, mas também, dos conhecimentos, atitudes e práticas sexuais de prevenção às DST e AIDS adquiridos pelos nossos pacientes ao longo dos anos de tratamento ambulatorial.

A diferença significativa encontrada entre os sexos na verdade está relacionada ao fato de que homossexuais masculinos foram à categoria de exposição mais freqüentemente acometida pela sífilis como observamos na análise multivariada. Tal achado corrobora os resultados dos trabalhos descritos anteriormente ${ }^{913}$.

Contudo, quando nos circunscrevemos aos nossos resultados de co-infecção; especulamos que a razão para o aparecimento de sífilis ativa em pacientes com HIV/AIDS sob cuidados médicos de longo prazo poderia estar na falsa impressão dada pelos avanços alcançados nos últimos anos com HAART que aumentou o tempo de sobrevida ${ }^{1015}$ dos pacientes e também a sua qualidade de vida. Com isto, alguns dos pacientes retornaram as suas rotinas habituais de vida o que inclui atividades sexuais, muitas vezes de risco. Similarmente, mas sob a perspectiva da mortalidade, observou-se diminuição da incidência da sífilis durante os anos de maior taxa de mortalidade da AIDS $^{4}$.

É preocupante o fato de que, em nosso estudo, 63,6\% dos casos de sífilis ocorreram em pacientes já haviam obtido cura da sífilis em tratamento anterior. Embora boa parte dos médicos possa acreditar que pacientes em acompanhamento rotineiro para HIV já conhecem os riscos relacionados às práticas sexuais desprotegidas, esse achado serve de alerta para que a adoção de medidas relacionadas ao sexo seguro deva ser um tema constantemente abordado nas consultas desses pacientes.

Devido ao fato de que a sífilis pode ter uma evolução mais rápida e mais agressiva nos pacientes portadores do HIV é fundamental que o diagnóstico dessa co-infecção seja feito o mais precoce possível com a realização de um rastreamento inicial, através do VDRL de todos os pacientes em acompanhamento.

Além disso, considerando-se que a co-infecção com outras DST, especialmente sífilis, aumenta a chance de transmissão do HIV e que muitos dos pacientes portadores do HIV continuam mantendo relações sexuais desprotegidas, o diagnóstico precoce desta coinfecção, em última análise, além de reduzir a chance de transmissão de síflis também reduz a chance de transmissão do HIV ${ }^{11}$.

\section{AGRADECIMENTOS}

Dario Hart Signorini por sua contribuição na montagem do banco de dados e uso dos programas de computação que formataram as referências deste manuscrito.

\section{REFERÊNCIAS}

1. Center for Disease Control and Prevention. HIV prevention through early detection and treatment of other sexually transmitted diseases-United States. Recommendations of the Advisory Committee for HIV and STD prevention. MMWR Recommendations and Reports 47 (RR-12): 1-24, 1998.

2. Center for Disease Control and Prevention. Primary and secondary syphilis-United States, 1997. MMWR Recommendations and Reports 47 (RR-24): 493-497, 1998. 
3. Center for Disease Control and Prevention. Incorporating HIV prevention into the medical care of persons living with HIV. Recommendations of CDC, the Health Resources and Services Administration, the National Institutes of Health, and the HIV Medicine Association of the Infectious Diseases Society of America. MMWR Recommendations and Reports 52 (RR-12): 1-24, 2003.

4. Chesson HW, Dee TS, Aral SO. AIDS mortality may have contributed to the decline in syphilis rates in the United States in the 1990s. Sexually transmitted diseases 305: 419-424, 2003.

5. Chesson HW, Heffelfinger JD, Voigt RF, Collins D. Estimates of primary and secondary syphilis rates in persons with HIV in the United States, 2002. Sexually transmitted diseases 325: 265-269, 2005.

6. Fonseca MG, Bastos FI, Derrico M, Andrade CL, Travassos C, Szwarcwald CL. [AIDS and level of education in Brazil: temporal evolution from 1986 to 1996]. Escola Nacional de Saúde Publica Fundação Oswaldo Cruz, Ministério da Saúde. Cadernos de Saúde Pública 16 (supl 1): 77-87, 2000.

7. Fonseca MG, Szwarcwald CL, Bastos FI. [A sociodemographic analysis of the AIDS epidemic in Brazil, 1989-1997]. Revista de Saúde Pública 366: 678-685, 2002.

8. Kilmarx PH, St Louis ME. The evolving epidemiology of syphilis. American Journal of Public Health 858 (part 1): 1053-1054, 1995.

9. Kyriakis KP, Hadjivassiliou M. HIV-1 infection-associated risk factors among sexually transmitted disease patients in Athens, Greece: 1990 to 1996. Sexually Transmitted Diseases 275: 259-265, 2000.

10. Marins JR, Barros MB, Machado H, Chen S, Jamal LF, Hearst N. Characteristics and survival of AIDS patients with hepatitis C: the Brazilian National Cohort of 1995-1996. AIDS (London, England) 19 (suppl): 4S27-30, 2005.
11. Minitério da Saúde. Epidemiologia. http://www.aids.gov.br/data/Pages/ LUMISBCD47A0DPTBRIE.htm, 2006

12. Passos MRL, Goulart Filho RA, Nascimento AVS, Barreto NA. Tratamento de sífilis adquirida com azitromicina. Jornal Brasileiro de Doenças Sexualmente Transmissíveis 13: 27-32, 2001.

13. Rodrigues EHG, Abath FGC. Doenças sexualmente transmissíveis em pacientes infectados com HIV/AIDS no Estado de Pernambuco, Brasil. Revista da Sociedade Brasileira de Medicina Tropical 331: 47-52, 2000

14. Santos NJS, Tayara Â, Silva SR, Buchalla CM, Laurenti R. A aids no Estado de São Paulo: as mudanças no perfil da epidemia e perspectivas da vigilância epidemiológica. Revista Brasileira Epidemiologia 53: 286-310, 2002.

15. Signorini DJHP, Codeço CT, Carvalho MS, Campos DP, Monteiro MCM, Andrade MFC, Pinto JFC, Sá CAM. Effect of sociodemographic, clinical-prophylactic and therapeutic procedures on survival of AIDS patients assisted in a Brazilian outpatient clinic. Revista Brasileira de Epidemiologia 8: 253-261, 2005.

16. Szwarcwald CL, Castilho EA, Barbosa Jr A, Gomes MR, Costa EA, Maletta BV, Carvalho RF, Oliveira SR, Chequer P. [Risk behavior among Brazilian Military conscripts, 1998: an study of HIV infections following socioeconomic differences]. Escola Nacional de Saúde Pública, Fundação Oswaldo Cruz, Ministério da Saúde. Cadernos de Saúde Pública 16 (supl 1): 113-128, 2000

17. Team RDC. R: A Language and Environment for Statistical Computing. Vienna, Austria: R Foundation for Statistical Computing, 2006 